reactivity levels and may lead to adverse cardiovascular outcomes in patients treated with drug eluting stents following cardiac catheterization. However, literature on omeprazole taken in association with clopidogrel is lacking in the neuroendovascular literature. We hypothesized that patients taking omeprazole would exhibit higher PRU levels and increased complications following treatment with a flow diverter device (FDD).

Methods All patients with a FDD placement for an intracranial aneurysm at a large tertiary institution from January 1st, 2015 to December 31st, 2018 were retrospectively analyzed. Inclusion criteria included 1) documented clopidogrel administration, 2) available P2Y12 levels, and 3) thorough documentation of administration of other medications including omeprazole. Outcomes analyzed included ischemic stroke on MRI and FDD stenosis on follow-up angiography.

Results Out of a total of of 138 patients that met the inclusion criteria, 16 (12\%) were taking both omeprazole and clopidogrel. The average age for the omeprazole patients was significantly higher than those not taking omeprazole[69( \pm 10$)$ vs 57 $( \pm 14) \quad(\mathrm{p}=0.001)]$. A significantly higher P2Y12 reactivity (decreased platelet inhibition) was observed in patients taking omeprazole $(\mathrm{PRU}=250)$ versus those not taking omeprazole $(P R U=110) \quad(p<0.001)$. Furthermore, a higher number of patients were found to have a P2Y12 level $>180$ PRU in the omeprazole $(\mathrm{N}=14,88 \%)$ vs no omeprazole $(\mathrm{N}=24,20 \%)$ patients $(\mathrm{p}<0.001$, OR 29; 95\% CI 6-134). There were no significant differences in the rates of ischemic strokes, FDD stenosis, or hemorrhagic complications between the two groups. Conclusion Omeprazole significantly increases the P2Y12 reactivity levels in intracranial aneurysm patients on clopidogrel treated with a FDD. However, omeprazole did not increase the risk of ischemic events and/or device stenosis. Nonetheless, given the significant association between omeprazole and decreased clopidogrel efficacy, omeprazole should not be administered to neuroendovascular patients treated with a FDD taking clopidogrel.

Disclosures J. Catapano: None. V. Fredrickson: None. A. Wakim: None. J. Lundberg: None. B. Hendricks: None. J. Baranoski: None. T. Cole: None. D. Wilkinson: None. N. Majmundar: None. F. Albuquerque: None. A. Ducruet: None.

\section{E-217 VOXEL BASED CALCULATION OF ANEURYSM VOLUME AND MORPHOLOGICAL CHARACTERISTICS}

C Settanni* ${ }^{*}$ T Becker, W Merrit. Northern Arizona University, Flagstaff, AZ

10.1136/neurintsurg-2020-SNIS.248

Introduction Various embolization techniques are available for intracranial aneurysms. The volume of an aneurysm is of interest for the device selection and delivery of a variety of embolization techniques, such as: coils, liquid embolics, flow disruptors, and flow diverters. Accurate aneurysm sizing and volumetric information can help interventionalists assess flow and stability pre-treatment, and assess potential remnant or recanalization risks post-treatment.

Materials and Methods This research project applies voxelbased volume calculations, from patient MRI medical imaging data, to determine accurate 3-D aneurysm volume calculations. Additionally, the application can display clinically relevant parameters, such as aneurysm neck diameter, dome height and midline-dome width (for dome: neck (D:N) ratio calculations. To develop the calculations, formalin-fixed canine aneurysms model samples are measured with a Bruker $7 \mathrm{~T}^{\circledR}$ MRI and reconstructed in 3-D. (figure 1).

The images is be processed using a MATLAB ${ }^{\circledR}$ algorithm. This algorithm patches together image segments from MRI and micro CT scans of animals and/or humans. The application acquires 3D MRI data, discretizes the domain intrasaccular aneurysm space, and evaluates the volume of the aneurysm sac. This data is compared to physical measurements of excised aneurysms and calibrated 2-D angiographic images.

Results Imaging processing techniques may be used to determine the domain of aneurysms with unprecedented precision, with less than $5 \%$ volumetric error, whereas current measurement techniques, especially in 2-D planes have errors as high as $30 \%$. This technique helps determine the macroscopic properties of aneurysms, as well as accurately calculate the volumes of heterogeneous features such as blebs or abnormal aneurysm shapes.

Conclusion This project brings together clinical and engineering expertise to translate medical imaging data directly into volumetric measurements with highly precise calculations that are currently not available from 2-D angiographic images. Measuring the size and dimensional properties of aneurysms with voxel-based volume calculations provides a fast, reliable and repeatable resource for aneurysm assessment. This technique will help interventionalists appropriately asses risk and treatment options for a broad variety of aneurysm morphologies.

Disclosures C. Settanni: None. T. Becker: 1; C; STTR (NIH). 2; C; Aneuvas. W. Merrit: 1; C; STTR(NIH). 2; C; Aneuvas.

\section{E-218 STENT-ASSISTED COILING OF ACUTE RUPTURED CEREBRAL ANEURYSMS}

V Berestov*, K Orlov, N Strelnikov, M Demyanovskaya. Neurosurgery department, National Medical Research Center (Meshalkin Clinic), Novosibirsk, Russian Federation

10.1136/neurintsurg-2020-SNIS.249


Abstract E-217 Figure 1 Left: 2D MRI longitudinal section of a surgically anastomosed canine sidewall aneurysm (1 month after creation), Middle: Decomposition of the 3D MRI by grouping values of equal brightness, Right: 3D reconstruction of the aneurysm sac 